\title{
Detecting Impulses in Mechanical Signals by Wavelets
}

\author{
W.-X. Yang \\ Institute of Vibration Engineering, Northwestern Polytechnical University, Xi'an 710072, China \\ Email: wen.yang@ntu.ac.uk
}

\author{
X.-M. Ren \\ Institute of Vibration Engineering, Northwestern Polytechnical University, Xi'an 710072, China \\ Email: renxmin@nwpu.edu.cn
}

Received 21 February 2003; Revised 17 October 2003; Recommended for Publication by Marc Moonen

\begin{abstract}
The presence of periodical or nonperiodical impulses in vibration signals often indicates the occurrence of machine faults. This knowledge is applied to the fault diagnosis of such machines as engines, gearboxes, rolling element bearings, and so on. The development of an effective impulse detection technique is necessary and significant for evaluating the working condition of these machines, diagnosing their malfunctions, and keeping them running normally over prolong periods. With the aid of wavelet transforms, a wavelet-based envelope analysis method is proposed. In order to suppress any undesired information and highlight the features of interest, an improved soft threshold method has been designed so that the inspected signal is analyzed in a more exact way. Furthermore, an impulse detection technique is developed based on the aforementioned methods. The effectiveness of the proposed technique on the extraction of impulsive features of mechanical signals has been proved by both simulated and practical experiments.
\end{abstract}

Keywords and phrases: wavelet transform, envelope analysis, fault diagnosis, rolling element bearing, soft threshold.

\section{INTRODUCTION}

The extraction of impulsive features in vibration signals is vital for diagnosing such machines as engines, rolling element bearings, gearboxes, and so on. Researchers have developed many methods for fulfilling this purpose, for example, cepstrum analysis [1], signal demodulation procedure [2], transmission error measurement [3], higher-order time-frequency analysis [4], moving window procedure [5], and envelope analysis [6]. These techniques either use a time domain averaging procedure or adopt the classical timefrequency analyzing method that only provides constant time/frequency resolution analysis, so they are not powerful enough to deal with nonstationary signals. Recently, interest in the use of wavelet transforms (WTs) for processing nonstationary signals has grown [7]. Different from these convenient methods, the WTs provide a constant frequency-tobandwidth ratio analysis. In consequence, WTs possess fine time resolution in the high frequency ranges and excellent frequency resolution in low frequency region. This feature of WTs uniquely fits the requirement in failure diagnosis [8]. However, the impulse detection results generated by WTs are still not easy to be identified especially when the signal-tonoise ratio (SNR) of the detected signal is low. In view of this, a new wavelet-based impulse detection technique is studied in this paper.

\section{SUPERIORITY OF MORLET WAVELET ON IMPULSE DETECTION}

The wavelet transform of a signal $x(t)$ is defined as

$$
\begin{aligned}
\mathrm{WT}_{x}(a, \tau) & =\left\langle\psi_{a, \tau}(t), x(t)\right\rangle \\
& =\frac{1}{\sqrt{a}} \int x(t) \psi^{*}\left(\frac{t-\tau}{a}\right) d t,
\end{aligned}
$$

where $\mathrm{WT}_{x}(a, \tau)$ represents the wavelet transforming coefficient derived from the signal $x(t)$ when setting the scale to be $a$ and the time shifting parameter to be $\tau$; the asterisk stands for complex conjugate; $\psi_{a, \tau}(t)$ the daughter wavelets of the mother wavelet $\psi(t)$, which is derived by varying both the scale factor $a$ and the shifting parameter $\tau$ continuously. The factor $1 / \sqrt{a}$ is used to ensure energy preservation.

From (1), it is found that the wavelet transform $\mathrm{WT}_{x}(a, \tau)$ is a function of the shifting parameter $\tau$ for each scale $a$. It manifests the information of $x(t)$ at different levels of resolution by measuring the similarity between the signal $x(t)$ and the daughter wavelet function $\psi_{a, \tau}(t)$ at different scales. This implies that the components of the signal may be extracted out perfectly when a wavelet function with similar shape as the component is employed. This is called the maximum matching mechanism adapted for WTs. In order to demonstrate this matching mechanism graphically, an 


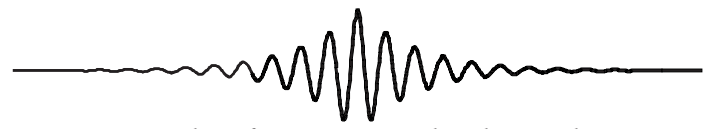

Impulsive feature contained in the signal
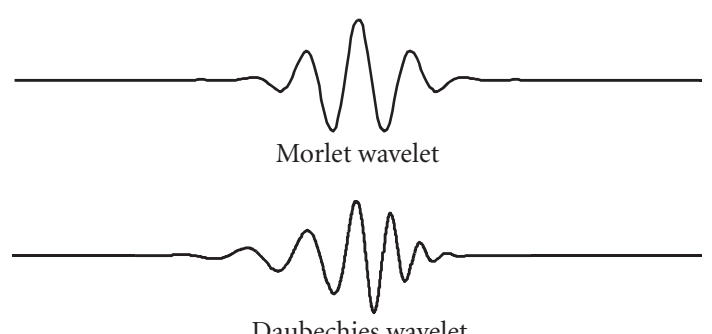

Daubechies wavelet

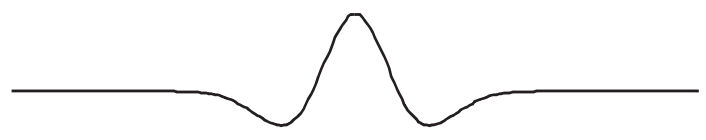

Mexican hat wavelet

(a)

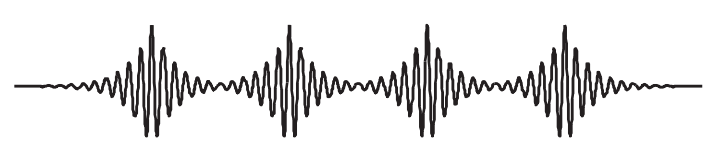

An arbitrary signal with periodic impulsive features

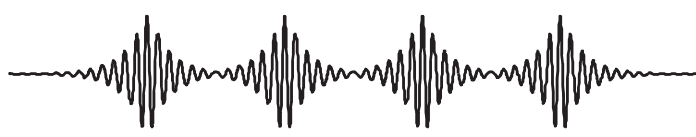

Coefficients generated by Morlet wavelet at scale 20

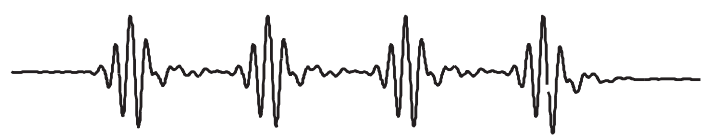

Coefficients generated by Daubechies wavelet at scale 20

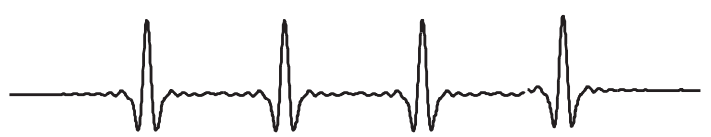

Coefficients generated by mexican hat wavelet at scale 20

(b)

FIGURE 1: Wavelet transforms of a simulated signal. (a) Impulsive feature and wavelets. (b) Signal and its wavelet transforming coefficients.

example is given in the following. Figure 1a shows a simulated impulsive feature usually contained in the signal and three kinds of wavelet functions (Morlet wavelet, Daubechies wavelet, and mexican hat wavelet) which are often used in practice. Figure $1 \mathrm{~b}$ shows a simulated signal with periodic impulsive features and its corresponding wavelet transforming coefficients derived at a scale of 20 by using Daubechies wavelet, mexican hat wavelet, and Morlet wavelet, respectively.

From Figure 1a, it was found that when compared to other two kinds of wavelet functions, the geometric shape of Morlet wavelet looks more like the impulsive feature contained in the signal. The results shown in Figure $1 \mathrm{~b}$ further demonstrate that, using Morlet wavelet, the impulsive features of the signal can be perfectly extracted. From this example, it is known that the selection of an appropriate wavelet function is actually a crucial work for guaranteeing the successful extraction of signal features.

The single freedom degree system subjected to an impact load may be formulated as

$$
M \frac{d^{2} x}{d t^{2}}+C \frac{d x}{d t}+K x=F \delta(t)
$$

where $x$ represents the displacement, $M$ the concentrated mass, $C$ the damping coefficient, and $K$ the stiffness of the system, $F$ is a constant, and

$$
\delta(t)= \begin{cases}1, & t=\tau \\ 0, & \text { otherwise. }\end{cases}
$$

The solution for (2) is

$$
\begin{aligned}
x(t)= & \frac{F+M v_{0}}{M \omega_{d}} e^{-\zeta \omega_{n} t} \sin \left(\omega_{d} t\right) \\
& +\frac{x_{0}}{\left(1-\zeta^{2}\right)^{1 / 2}} e^{-\zeta \omega_{n} t} \cos \left(\omega_{d} t-\psi\right),
\end{aligned}
$$

where $\omega_{n}=\sqrt{K / M}, \zeta=C / 2 M \omega_{n}, \omega_{d}=\omega_{n} \sqrt{1-\zeta^{2}}$, the phase angle $\psi=\tan ^{-1}\left(\zeta / \sqrt{1-\zeta^{2}}\right)$, and $x_{0}$ and $v_{0}$ indicate the initial displacement and velocity of the system, respectively.

When the initial displacement and velocity of the system are zero, that is, $x_{0}=0, v_{0}=0,(4)$ can be rewritten as

$$
x(t)=A e^{-\zeta \omega_{n} t} \sin \left(\omega_{d} t\right),
$$

where $A=F / M \omega_{d}$.

Equation (5) indicates that the impulsive feature, which is caused by external impact load, is characterized by an oscillation with decaying amplitude. So according to the matching mechanism of wavelet transform that has been proved in Figure 1, Morlet wavelet could be a more suitable wavelet function for extracting such types of features, because Morlet wavelet has a more similar shape to the impulsive feature. The complex Morlet wavelet function can be expressed as

$$
\begin{aligned}
\psi(t) & =\frac{1}{\sqrt{2 \pi}} e^{-\left(t^{2} / 2\right) \beta^{2}}[\cos (\omega t)+j \sin (\omega t)] \\
& =\frac{1}{\sqrt{2 \pi}} e^{-\left(t^{2} / 2\right) \beta^{2}} e^{j \omega t} .
\end{aligned}
$$

Actually, (6) is similar to (5) in both structure and compositions. In addition, it is noticed from (6) that the parameter $\beta$ determines the geometric shape of Morlet wavelet. When $\beta$ tends to zero, the function tends to a cosine function which has fine frequency resolution, and when $\beta$ tends to $+\infty$, the function inclines to be an impulse function and its time resolution will be increased notably. So it is natural to expect that the Morlet wavelet with larger $\beta$ is suitable to extract impulses in mechanical signals. It is necessary to note that, in order to satisfy the admissibility condition of the wavelet [9] and guarantee that the modified Morlet wavelets are always "band-pass" filters, the parameter $\beta$ should not be adjusted 
arbitrarily. This limitation seems to have been omitted by $[10]$.

It is well known that, in the frequency domain, the impulse has response in the whole frequency region, while the harmonic signals have response only in a narrow band of frequency. This suggests that we may detect impulses by performing WTs in one special frequency region, where the harmonic signals have little or no response, but the impulse response is still significant. Since the time resolution of wavelet transform notably increases depending on the duration of the mother wavelet, the nonstationary feature (including impulse), in most cases, can be better revealed if the wavelet transform is carried out in the high frequency region.

Therefore, two measures may be taken for impulse detection. The first is to properly adjust the shape control parameter $\beta$ of the Morlet wavelet function, the second is to perform the WTs at a special frequency region in which the harmonic signals have little or no response, but the inspected impulse still has strong response.

\section{ENVELOPE ANALYSIS BASED ON COMPLEX MORLET WAVELET}

In the past, the envelope analysis of the signal was carried out with the aid of the Hilbert transform. In essence, the Hilbert transform can be considered to be a filter that simply shifts phases of all frequency components of its input by $-\pi / 2$ radians. $x(t)$ and $y(t)$ form the complex conjugate pair of an analytic signal $z(t)$ as

$$
z(t)=x(t)+i y(t)=A(t) e^{i \theta(t)}
$$

with $A(t)=\left[x(t)^{2}+y(t)^{2}\right]^{1 / 2}, \theta(t)=\tan ^{-1}[y(t) / x(t)]$. Where $i=\sqrt{-1}$, the time-varying function $A(t)$ is the so-called instantaneous envelop of the signal $x(t)$, which extracts the slow time variation of the signal.

Because the Hilbert spectrum uses a transform rather than convolution as in the Fourier analysis, the practice demonstrates that for a transient signal, the Hilbert spectrum does offer clearer frequency-energy decomposition than the traditional Fourier spectrum. However, during the implementation of the Hilbert transform, it deals with different frequency components without any distinguishing. Moreover, from (7), it is found that the computation of $y(t)$ still requires the knowledge of $x(t)$ for all values of $t$. Thus, the "local" property of the Hilbert transform is in fact a "global" property of the signal. In view of these reasons, the complex wavelet-based envelope detection method is designed for extracting the impulsive features contained in the signals.

The complex Morlet wavelet transform of a modulated signal $x(t)$ can be written as

$$
\mathrm{WT}_{x}(a, \tau)=\frac{1}{\sqrt{2 \pi a}} \int x(t) e^{-\left([(t-\tau) / a]^{2} / 2\right) \beta^{2}} e^{[j \omega(t-\tau) / a]} d t .
$$

Since the complex Morlet wavelet is adopted in (8), all wavelet transforming coefficients $\mathrm{WT}_{x}$ derived by (8) are complex numbers. Similar to the Hilbert-transform-based

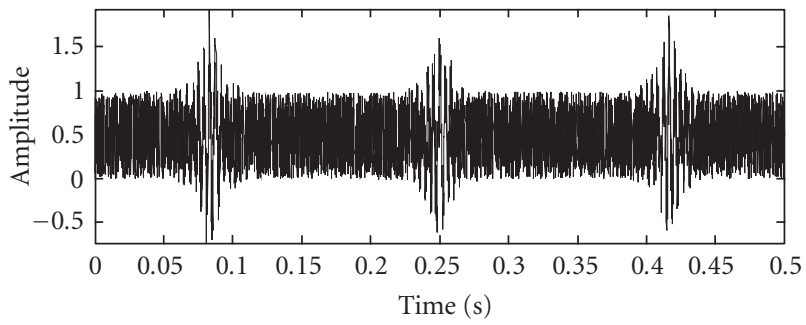

(a)

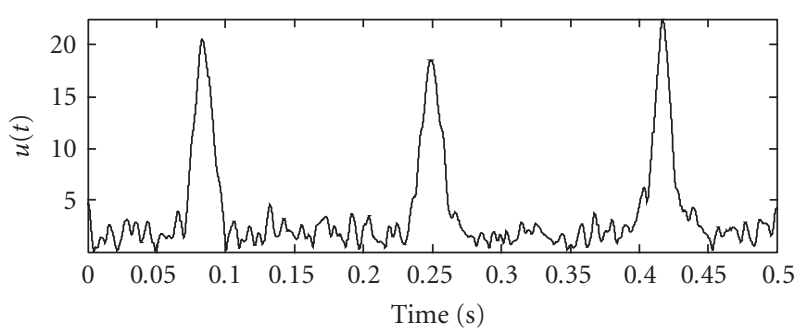

(b)

FIGURE 2: Distinguishing impulses from simulated noisy signal. (a) Noisy impulse signal. (b) Envelope analysis of the noisy impulse signal.

envelope analysis, the wavelet-based envelope analysis is performed by

$$
u(t)=\sqrt{\left\{\operatorname{Re}\left[\mathrm{WT}_{x}(a, t)\right]\right\}^{2}+\left\{\operatorname{Im}\left[\mathrm{WT}_{x}(a, t)\right]\right\}^{2}},
$$

where $u(t)$ indicates the envelope analysis results.

In the following, a noisy impulse-contained signal was employed for verifying the effectiveness of this new envelope analysis method. Figure 2 a shows the original noisy signal with impulsive features, and Figure $2 \mathrm{~b}$ shows its corresponding envelope analysis result $u(t)$. Where $\mathrm{SNR}=1.0, \beta=1$, the wavelet transform is performed at frequency $400 \mathrm{~Hz}$.

It can be clearly seen from Figure 2 that the envelopes of the signal are extracted out perfectly. Additionally, as Morlet wavelet itself is one kind of bandpass filter, the proposed method has a good capacity for noise reduction. The noise in the analyzed results is suppressed to a certain extent, so that the impulses in the derived results become more explicit and more easily identified. This method will undoubtedly facilitate the machine fault diagnosis.

However, the wavelet-based envelope analysis on its own is not sufficient to reduce noise and highlight the interesting features contained in the signals. It has been reported that using the "soft-thresholding" method [10] can further enhance this function. Hence, a new flexible soft-threshold-based denoising method is further studied in the following section.

\section{SOFT THRESHOLD}

The application of threshold criterion is effective in reducing noise and highlighting the interesting features in mechanical 


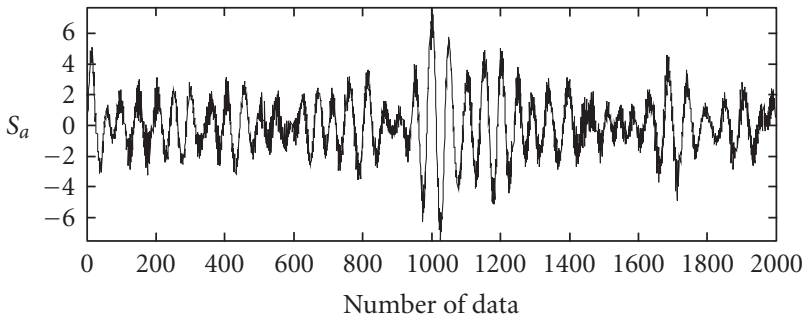

(a)

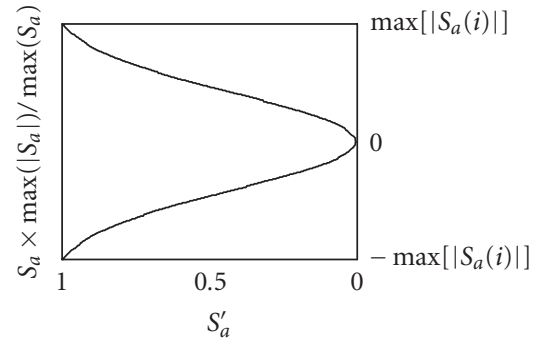

(b)

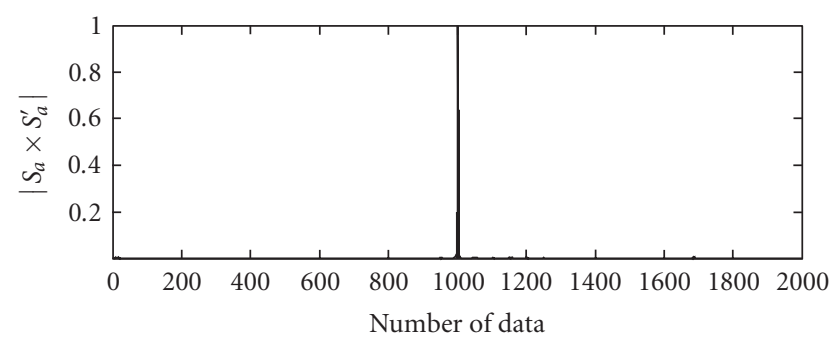

(c)

Figure 3: Working mechanism of the new soft-threshold function. (a) $S_{a}$; (b) Soft-threshold function; (c) $\left|S_{a} \times S_{a}^{\prime}\right|$.

signals [10], but how to choose an ideal threshold still remains an unanswered question. Here, a new soft threshold function is designed for making the denoising process more adaptive and smoother. It is

$$
S_{a}^{\prime}(i)=e^{-\xi \times\left\{\max \left[\left|S_{a}(i)\right|\right]-\left|S_{a}(i)\right|\right\}^{2}},
$$

where $S_{a}^{\prime}(i)$ represents the relative value of the $i$ th coefficient at scale $a,\left.S_{a}(i)\right|_{i=1, \ldots, N}$ the $i$ th coefficient, and $\xi>0$ the decay parameter. It is easy to know that the larger the value of $\xi$, the faster the function decays. The working mechanism of this soft-threshold function is as illustrated in Figure 3, where the value of $\xi$ is taken to be 3 . By multiplying the wavelet coefficients $S_{a}$ with the soft-threshold function $S_{a}^{\prime}$, the results derived by wavelet transform may be further purified. The purified results are shown in Figure $3 c$.

From (10), it was found that the larger the value of $\xi$, the faster the function decays. In other words, when $\xi$ approaches to a large value, only a few number of data that are very close to the $\max \left[\left|S_{a}\right|\right]$ are retained, while most data are suppressed. Consequently, the impulsive feature in mechanical signals is highlighted in this case. On the contrary, when $\xi$ approaches a small value, more data are preserved and only a few number of data with small values are suppressed. This case is more suitable for processing harmonic signals. Obviously, with the aid of adjustable $\xi$, the proposed soft threshold is more adaptive for feature extraction. Besides, this soft threshold has another merit, that is, however much the data $S_{a}$ is suppressed, its relative value $S_{a}^{\prime}$ will not be zero. This is distinctly different from many other available threshold criteria $[11,12]$. So, in comparison, the new soft threshold can lead to a much smoother result. The value of $S_{a}^{\prime}$ generated by the new soft threshold is limited to the half-closed region $(0,1]$.

\section{DEVELOPMENT OF THE IMPULSE DETECTION METHOD}

looseness $=1$ Based on the techniques proposed above, an advanced impulse detection strategy is developed, as depicted in Figure 4.

It is necessary to note that, during the implementation of this strategy, the parameters $\beta$ and $\xi$ as well as the scale $a$ should be selected appropriately according to the practical situation of the inspected signals. Often a satisfactory impulse detection result can be obtained when the parameter $\beta$ is taken to be a larger one. But it should be kept in mind that it should not be adjusted arbitrarily so that the admissibility condition of the wavelet [9] is satisfied. WTs are used at high frequencies to detect shock impulses in signals measured from rolling element bearings. However, when diagnosing gearbox vibration, the impulses generated due to tooth breakage may be identified more satisfactorily if the WTs are performed at frequencies lower than the meshing frequency of the gear couple. In practical applications, appropriate scale region of WTs can be selected by using the method given in [13].

To verify the effectiveness of the strategy, a simulated experiment was performed first. The raw signal and the impulse detection results obtained in the high frequency region $[0.4 \mathrm{kHz}, 2.0 \mathrm{kHz}]$ are shown in Figure 5 , where $\beta=5.6$, $\xi=6$. 


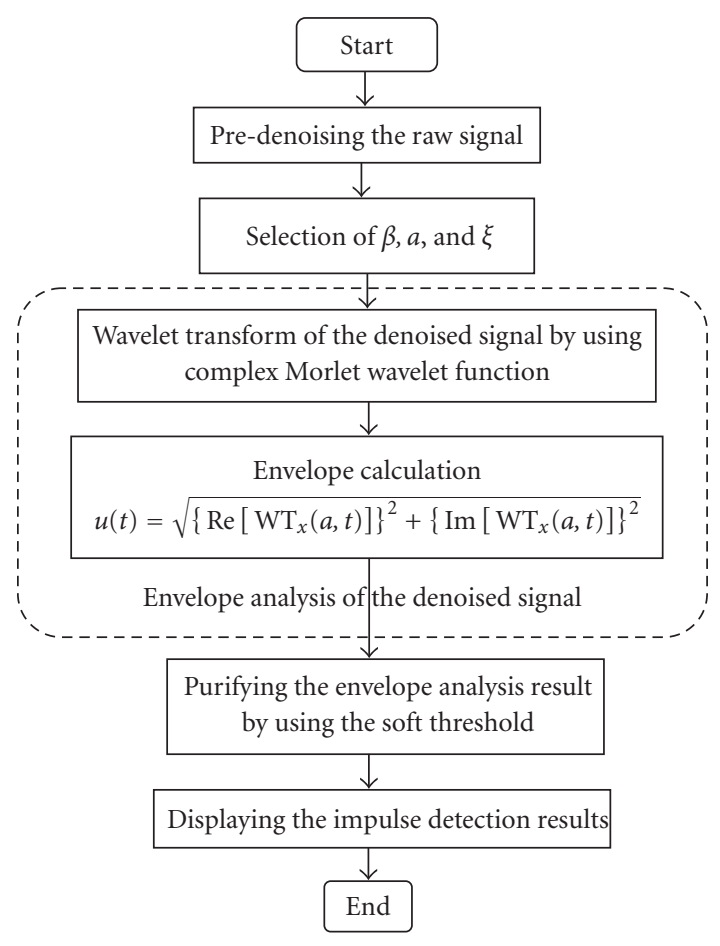

FIgURE 4: Flow chart of the impulse detection method.

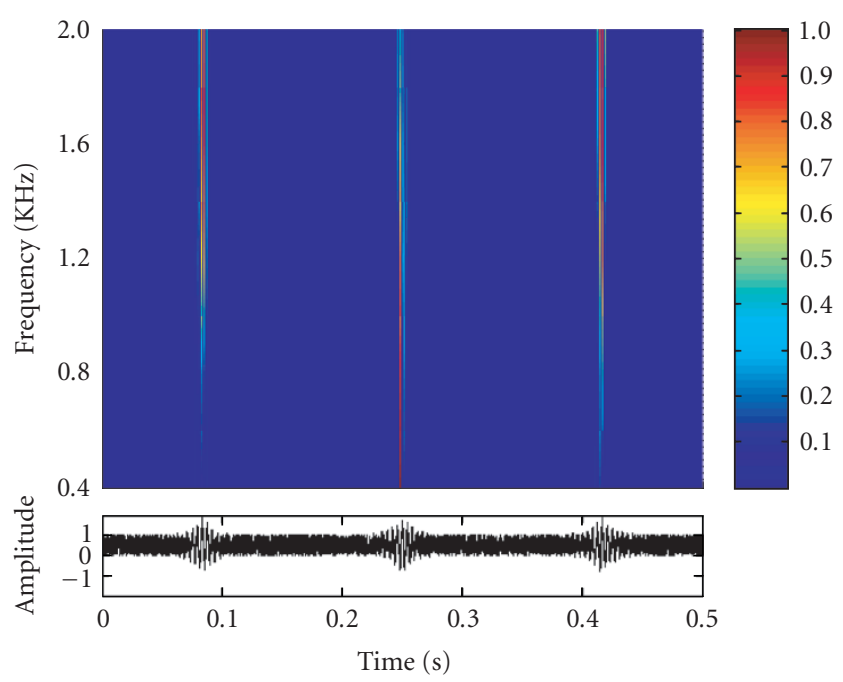

Figure 5: Detecting impulses from the noisy signal using the impulse detection method depicted in Figure 4.

Figure 5 suggests that the proposed strategy is actually effective at impulse detection even if the inspected signal is heavily polluted by background noise. In the following, the strategy to deal with the vibratory signals collected from a ball bearing with ball flaw fault was applied. The geometric parameters of the bearing are listed in Table 1 .

Using these parameters, the characteristic frequencies corresponding to different bearing faults were calculated us-
TABLE 1: Geometric parameters of the bearing.

\begin{tabular}{ll}
\hline Ball diameter & $d=7.5 \mathrm{~mm}$ \\
Pitch diameter & $D=39.45 \mathrm{~mm}$ \\
Contact angle & $\alpha=5 \backsim 20^{\circ}$ \\
Number of rolling element (with two rows) & $n=2 \times 13=26$ \\
\hline
\end{tabular}

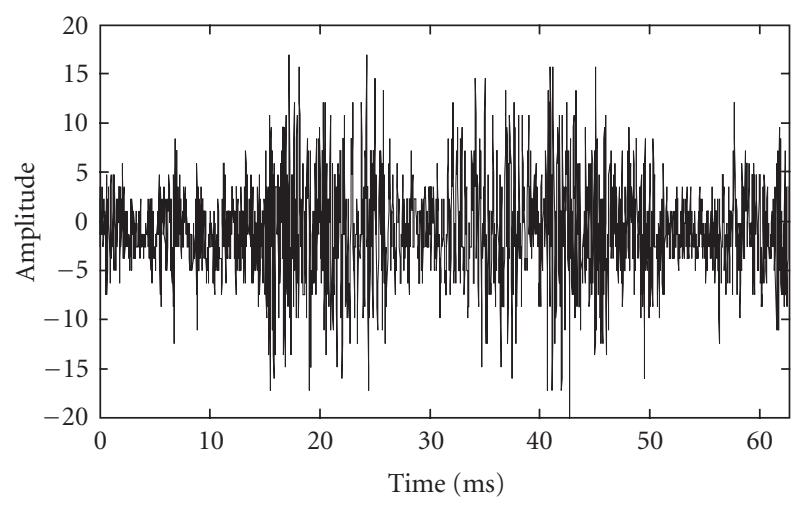

Figure 6: Vibration signals of the ball bearing.

ing the following equations [14]:

$$
\begin{aligned}
& f=\frac{n}{2} f_{r}\left(1-\frac{d}{D} \cos \alpha\right) \quad \text { for outer race failure, } \\
& f=\frac{n}{2} f_{r}\left(1+\frac{d}{D} \cos \alpha\right) \quad \text { for inner race failure, } \\
& f=\frac{D}{d} f_{r}\left[1-\left(\frac{d}{D}\right)^{2} \cos ^{2} \alpha\right] \quad \text { for rolling element failure, }
\end{aligned}
$$

where $f$ stands for the characteristic frequency corresponding to different kinds of faults, $f_{r}=25 \mathrm{~Hz}$ the relative rotating frequency between the inner and the outer races, $n$ the number of rollers (balls), $\alpha$ the contact angle between the race and the roller, $d$ the roller diameter, and $D$ the pitch diameter of the bearing. The theoretical characteristic failure frequencies calculated by (11) are between $263-267 \mathrm{~Hz}$ for outer race failure, $383-387 \mathrm{~Hz}$ for inner race failure, and are $127 \mathrm{~Hz}$ for a ball flaw fault, respectively. The vibration signal collected from the ball bearing is shown in Figure 6 .

Let $\beta=5.8, \xi=6$, and by performing the complex Morlet wavelet transform in the frequency region from 500 to $6500 \mathrm{~Hz}$, the results analyzed are shown in Figure 7.

It can be clearly seen from Figure 7 that some successive impulses are present in the whole frequency region. Moreover, a time interval approximating to 8.6 milliseconds (corresponding to a frequency value of $116 \mathrm{~Hz}$ ) was found between adjacent impulses, which was close to the characteristic frequency of $127 \mathrm{~Hz}$ for a ball flaw fault. It was suspected therefore that a ball fault had occurred in the bearing being inspection.

In order to confirm this prediction, a physical inspection 


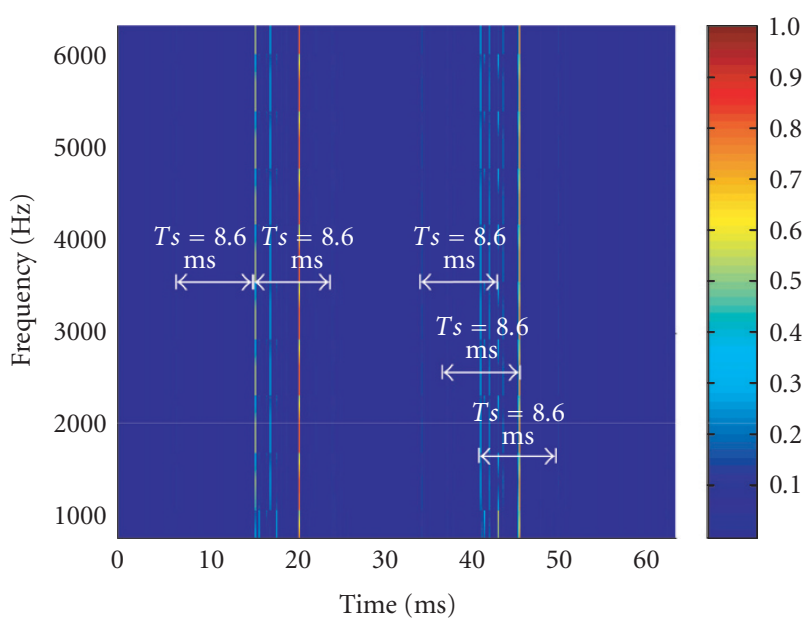

FIGURE 7: Analyzed result of the signal shown in Figure 5.

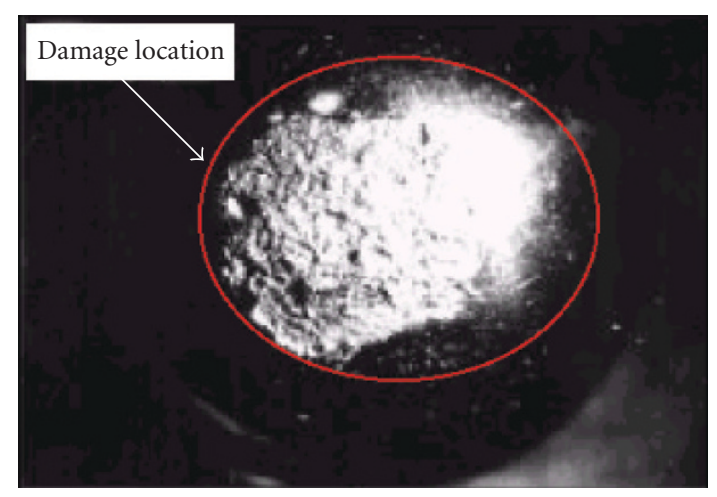

FIGURE 8: The damaged ball found in the inspected bearing.

of the bearing was undertaken and a damaged ball was found. It is as shown in Figure 8.

\section{CONCLUSIONS}

The above theoretical analysis and discussions lead to following conclusions.

(1) After processing the data using the proposed waveletbased envelope analysis method, the impulses buried in the noisy signals have been identified for further analysis. In the analyzed results, the impulsive features become more explicit and much easier to identify, thus effectively facilitating the diagnosis of machine faults.

(2) With the aid of the adjustable decay parameter, the proposed soft-threshold function is more adaptive for feature extraction and can lead to a smoother result. It overcomes the rigid performance of available hard/soft-threshold criteria.

(3) The advanced impulse detection technique developed based on the proposed wavelet-based envelope analysis method and the new adaptive soft-threshold function is effective at extracting the impulsive features from those mechanical signals with low SNR.

\section{ACKNOWLEDGMENTS}

The work described in this paper was supported by the National Natural Science Fund of China (Ref. No. 50205021) and the Shaanxi Provincial Natural Science Fund (Ref. No. $2002 \mathrm{E}_{2} 26$ ). The authors would like to express their special appreciation to reviewers and Dr. M. D. Seymour. Their kind suggestions significantly improved the quality of the paper.

\section{REFERENCES}

[1] R. B. Randall, "Cepstrum analysis and gearbox fault diagnosis," Tech. Rep. 13-150, Bruel \& Kjaer Application Notes, Denmark, 1981.

[2] C. Cempel and W. J. Staszewski, "Signal demodulation techniques in vibroacoustical diagnostics of machinery," Machine Dynamics Problems, vol. 2, pp. 161-174, 1991.

[3] W. J. Wang, Gearbox condition monitoring and early damage diagnosis by two and three dimensional vibration signal analysis, Ph.D. thesis, Department of Engineering Science, Oxford University, 1993.

[4] S. K. Lee and P. R. White, "Higher-order time-frequency analysis and its application to fault detection in rotating machinery," Mechanical Systems and Signal Processing, vol. 11, no. 4, pp. 637-650, 1997.

[5] W. J. Staszewski and G. R. Tomlinson, "Local tooth fault detection in gearbox using a moving window procedure," $\mathrm{Me}$ chanical Systems and Signal Processing, vol. 11, no. 3, pp. 331350, 1997.

[6] R. Rubini and U. Meneghetti, "Application of the envelope and wavelet transform analyses for the diagnosis of incipient faults in ball bearings," Mechanical Systems and Signal Processing, vol. 15, no. 2, pp. 287-302, 2001.

[7] P. Vandergheynst, J.-P. Antoine, E. Van Vyve, A. Goldberg, and I. Doghri, "Modeling and simulation of an impact test using wavelets, analytical solutions and finite elements," International Journal of Solids and Structures, vol. 38, pp. 5481-5508, 2001.

[8] W. J. Wang, "Wavelets for detecting mechanical faults with high sensitivity," Mechanical Systems and Signal Processing, vol. 15, no. 4, pp. 685-696, 2001.

[9] I. Daubechies, Ten Lectures on Wavelets, vol. 61 of CBMS-NSF Regional Conference Series in Applied Mathematics, SIAM, Philadelphia, Pa, USA, 1992.

[10] D. L. Donoho, "De-noising by soft-thresholding," IEEE Transactions on Information Theory, vol. 41, no. 3, pp. 613-627, 1995.

[11] J. Lin and L. S. Qu, "Feature extraction based on Morlet wavelet and its application for mechanical fault diagnosis," Journal of Sound and Vibration, vol. 234, no. 1, pp. 135-148, 2000.

[12] P. Tse, G. Xu, L. S. Qu, and S. Kumara, "An effective and portable electronic stethoscope for fault diagnosis by analysing machine running sound directly," International Journal of Acoustics and Vibration, vol. 6, no. 1, pp. 23-31, 2001.

[13] N. Delprat, B. Escudie, P. Guillemain, R. Kronland-Martinet, P. Tchamitchian, and B. Torresani, "Asymptotic wavelet and Gabor analysis: extraction of instantaneous frequencies," IEEE Transactions on Information Theory, vol. 38, no. 2, pp. 644-664, 1992.

[14] P. Tse, Y. H. Peng, and R. Yam, "Wavelet analysis and envelop detection for rolling element bearing fault diagnosis - their effectiveness and flexibility," Transactions of the ASME: Journal of Vibration and Acoustics, vol. 123, no. 3, pp. 303-310, 2001. 
W.-X. Yang got his Ph.D. degree from Xi'an Jiaotong University in 1999. Now, he is an Associate Professor working in Northwestern Polytechnical University, Xi'an, China. He majors in signal processing, nondestructive detection, machine condition monitoring, fault diagnosis, and other related researches.

X.-M. Ren got his Ph.D. degree from Northwestern Polytechnical University in 1999. Now he is the Head of the Institute of Vibration Engineering in this university. He majors in vibration analysis, dynamics, signal processing, and automatic control.
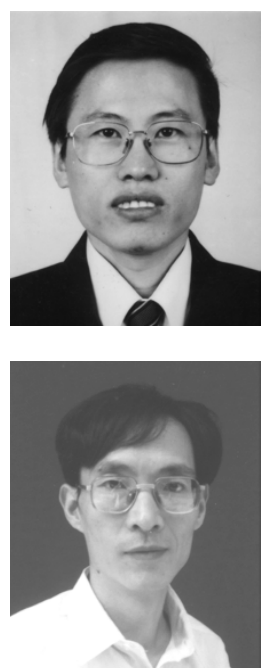